\title{
OPA1 alternate splicing uncouples an evolutionary conserved function in mitochondrial fusion from a vertebrate restricted function in apoptosis
}

\author{
A Olichon $^{1,3}$, G ElAchouri ${ }^{2}$, L Baricault ${ }^{1}$, C Delettre ${ }^{2}$, P Belenguer ${ }^{1}$ and G Lenaers ${ }^{\star, 1,2}$
}

In most eucaryote cells, release of apoptotic proteins from mitochondria involves fission of the mitochondrial network and drastic remodelling of the cristae structures. The intramitochondrial dynamin OPA1, as a potential central actor of these processes, exists as eight isoforms resulting from the alternate splicing combinations of exons (Ex) $4,4 \mathrm{~b}$ and $5 \mathrm{~b}$, which functions remain undetermined. Here, we show that Ex4 that is conserved throughout evolution confers functions to OPA1 involved in the maintenance of the $\Delta \Psi_{\mathrm{m}}$ and in the fusion of the mitochondrial network. Conversely, Ex4b and Ex5b, which are vertebrate specific, define a function involved in cytochrome $c$ release, an apoptotic process also restricted to vertebrates. The drastic changes of OPA1 variant abundance in different organs suggest that nuclear splicing can control mitochondrial dynamic fate and susceptibility to apoptosis and pathologies.

Cell Death and Differentiation (2007) 14, 682-692. doi:10.1038/sj.cdd.4402048; published online 6 October 2006

Mitochondrial membrane dynamics, involving both the fusionfission of the mitochondrial network and remodelling of cristae structures, are essential processes in cellular adaptation to changes of growth condition and programmed cell death. ${ }^{1-3}$ The outer and inner mitochondrial membrane (OMM and IMM) dynamics crosstalk and involve large GTPases: respectively, the mitofusins $M f n 1$ and $\mathrm{Mfn}^{4,5}$ and the dynamin-related $\mathrm{DRP}^{6}{ }^{6}$ on the OMM, and the dynaminrelated OPA1, localized in the inter membrane space (IMS). ${ }^{7}$ Mitochondrial fission is tightly associated to the apoptotic process, and physical and functional interactions between apoptotic proteins including Bcl2-homologue domains and DRP1 and mitofusins have been identified. ${ }^{8}$ In addition, $\mathrm{BH} 3-$ only proapoptotic proteins, exemplified by tBid and Bik, might act directly on the IMM structure, although their intramitochondrial target remains unknown. ${ }^{3,9}$ Further crosstalk between the OMM and the IMM is exemplified by the OMM fission and fusion regulation by the mitochondrial membrane potential $\left(\Delta \Psi_{\mathrm{m}}\right)^{10}$ and structural integrity ${ }^{11}$ and by interaction between OPA1 and Mfn1. ${ }^{12}$

The IMM forms, an architecturally complex structure, that has been reconsidered recently in the light of tomography analysis associated to transmission electron microscopy (TEM). Indeed, tubular inner membrane junctions, called cristae junctions, have been evidenced between the IMM facing the OMM and the vesicular-shaped cristae. ${ }^{13}$ In vertebrate cells, during the apoptotic process, the cristae junction topology is drastically modified to mobilize cyto- chrome c (cytc) from the intracristae compartment to the IMS, before its extensive release in the cytoplasm. ${ }^{3,9}$ How this process is physically controlled and related to the OMM dynamic remains unknown. We and others have shown that OPA1 is a good candidate for the regulation of the cristae structure in the mitochondria. ${ }^{11,14}$ Indeed, downregulation of OPA1 affect the IMM structure and integrity, leading to respiration defects and $\Delta \Psi_{\mathrm{m}}$ loss, fission of the mitochondrial network, impairment of growth and apoptosis ${ }^{11,14-18}$ and OPA1 overexpression protects from cyt $c$ mobilization by tightening cristae junctions. ${ }^{14}$ OPA1 is also partially associated to the OMM, on its IMS side, where it might interact with Mfn1 to promote fusion of the mitochondrial network, or maintain a physical link between the OMM and IMM. ${ }^{12,19}$ The different localization of OPA1 in the IMS and the multiple functions associated to this protein fit with the existence of 2-5 isoforms, evidenced by Western blot in different cell lines. They might result from alternate splicing of exons (Ex) 4, 4b and $5 \mathrm{~b}$ in OPA $1 \mathrm{mRNA}^{20}$ and from intramitochondrial-specific processing. ${ }^{21,22}$ Indeed, two intramitochondrial proteases, the rhomboid-like PARL and the m-AAA Paraplegin, are involved in OPA1 proteolytic cleavage, either to control the apoptotic process or the mitochondrial morphology. ${ }^{21,22}$ These cleavages are partial in normal growth condition and occur in peptides corresponding to Ex5 and Ex5b; consequently, they generate long and short forms for each OPA1 isoform. ${ }^{21}$ Together, these data suggest that OPA1 amino-terminal domain, unique among the dynamin family, is critical to

\footnotetext{
${ }^{1}$ Laboratoire de Biologie Cellulaire et Moléculaire du Contrôle de la Prolifération, CNRS UMR 5088, Université Paul Sabatier, 118 route de Narbonne, Toulouse, Cedex, France and ${ }^{2}$ INSERM U583, Montpellier, France and Institut des Neurosciences de Montpellier, IFR76, Université de Montpellier, BP 74103, Montpellier, Cedex, France ${ }^{*}$ Corresponding author: G Lenaers, INSERM U583, Institut des Neurosciences de Montpellier, BP 74103, Montpellier Cedex 5, France. Tel: + 33 499 636053 ; Fax: + 334996360 20; E-mail: glen@montp.inserm.fr

${ }^{3}$ Current address: EMBL, Meyerhofstrasse 1, Heidelberg 69117, Germany

Keywords: OPA1; alternate splicing; mitochondria; fusion; apoptosis

Abbreviations: ADOA, autosomal-dominant optic atrophy; cyt $c$, cytochrome $c ; \Delta \Psi_{\mathrm{m}}$, mitochondrial membrane potential; IF, immunofluorescence; IMM, inner mitochondrial membrane; IMS, inter membrane space; OMM, outer mitochondrial membrane; Q-PCR, quantitative polymerase chain reaction; TEM, transmission electron microscopy

Received 23.12.05; revised 07.8.06; accepted 11.9.06; Edited by JC Martinou; published online 6.10.06
} 
specify functional and regulatory mechanisms. In addition, among the almost 100 mutations, spanning the 31 exons of OPA1 (eOPA1 database), ${ }^{23}$ identified in autosomal-dominant optic atrophy patients (MIM165500), none involve Ex4b and Ex5b, suggesting that their integrity is a strict requisite for OPA1 functions. Here, we investigate the involvement of the alternate spliced exons in specifying OPA1 functions.

\section{Results}

Molecular characteristics of OPA1 mRNA variant and protein isoform sequences. OPA1 and its orthologues, encoded by a single nuclear gene, are the only proteins belonging to the dynamin family that are located inside mitochondria. ${ }^{7,24,25}$ In yeast, Mgm1 appears in Western blots as doublet, the fast migrating form resulting from the cleavage by a mitochondrial rhomboid-like protease of the slow migrating form. ${ }^{26,27}$ In human and mouse cell lines, previous works have reported the presence of 2-5 different electrophoretic forms of the OPA1 protein, ${ }^{7,11,19}$ which also result from different proteolytic cleavages. ${ }^{21,22}$ This contrasts with the existence of eight alternative splicing mRNAs that result from the inclusion or the exclusion of Ex4, Ex4b and Ex5b in the open reading frames (Figure 1a). ${ }^{20}$ Amino-acid sequence alignment between human OPA1 and orthologous a

$\begin{array}{cl}\begin{array}{c}\text { mRNA } \\ \text { variant }\end{array} & \begin{array}{c}\text { protein } \\ \text { isoforms }\end{array} \\ 2 & \text { OPA 1-0 } \\ 1 & \text { OPA 1-4 } \\ 3 & \text { OPA 1-4b } \\ 4 & \text { OPA1-5b } \\ 5 & \text { OPA 1-4.4b } \\ 7 & \text { OPA 1-4.5b } \\ 6 & \text { OPA 1-4b.5b } \\ 8 & \text { OPA1-4.4b.5b }\end{array}$

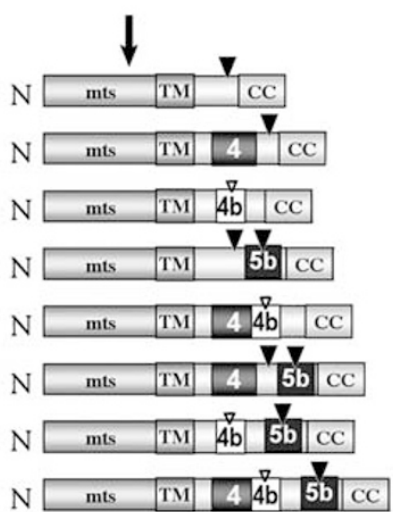

Domain 4

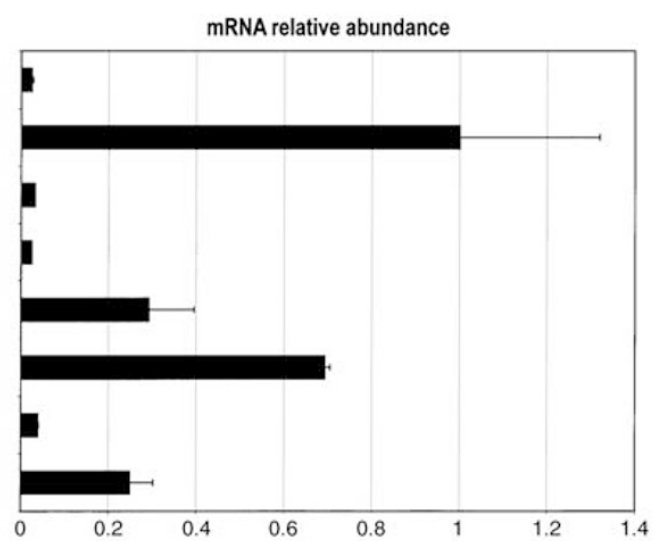

C
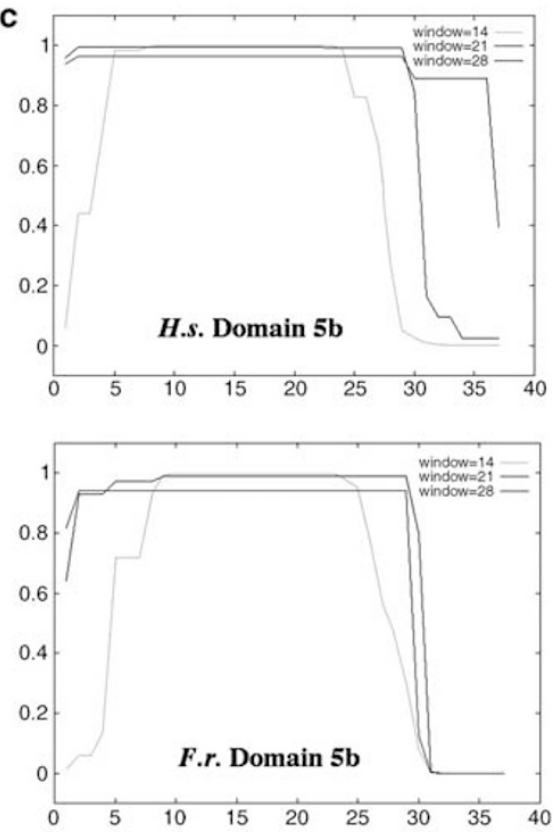

H.S. KIRKALPNSEDLVKLAPDFDKI-VESLSLLKDFFTSG

M.m. KIRKALPSSEDLASLAPDLDKI-TESLSLLKDFFTAG

D.r. KLASALPELEEIAKLLPDMEKI-GENFTFLKSLLSSE

D.m. QGERWSQFSRNLIEVGSL-VKNAIEVDPKLKQLGEDK

C.e. SNGSWNEFSQKM----KG-IKDGFGAD------GQNK

S.c. KMEEASSFTKDK----LDRIKDLGESM----KEKFNK

S.p. QVQRASDYTKDI------FDKTFGILD-----STWEK

\section{Domain $4 \mathrm{~b}$}

$\begin{array}{ll}\text { H.S. HKLVSEVIGASDLLLLL } \\ \text { M.m. } & \text { PLVSEVLEVSEALLLL } \\ \text { R.n. } & \text { KLVSEVIEASE LLLL } \\ \text { D.r. } & \text { TGLGSEVNGAS } L R L L L \\ \text { F.r. } & \text { VSLVSEVKGASGL LLL }\end{array}$

\section{Domain $5 b$}

H.S. GLLGELILLQQQIQEHEEEARRAAGQYSTSYAQQKRK M.m. GLLGELILLQQQIQEHEEEARRAAGQYSTSYAQQKRK R.n. GLLGELILLQQQIQEHEEEARRAAGQYSTSYAQQKRK

D.r. GLLSELIVIQQQIQ HEEEMRAAA-NNAPPPPRDPS F.r. GLLGELILIQQQIQRHEEE RRAAAANNARPPPPEPA

Figure 1 Schematic representation and sequence analysis of human OPA1 variants. (a) Schematic representation of primary structures of the eight OPA1 isoform aminoterminal regions upstream of the GTPase domain. The arrow indicates the predicted mitochondrial presequence cleavage sites conserved for all isoforms. ${ }^{7,21}$ The black arrowheads show possible cleavage sites in domains 5 and $5 \mathrm{~b}$ as proposed by Ishihara et al. ${ }^{21}$ The triangles show an eventual cleavage site in domain $4 \mathrm{~b}$. mRNA variant numbering corresponds to PubMed classification. Histogram represents the relative amount of OPA1 variant mRNAs in HeLa cells. (b) Evolutionary conservation of domains 4, 4b and 5b. Amino-acid alignments of domains 4, $4 \mathrm{~b}$ and $5 \mathrm{~b}$ from OPA1 orthologues in Hs, homo sapiens; Rt, Ratus norvegicus; Mm, mus musculus; Dr, Danio rerio; Fr, Fugu rubripes; D.m., Drosophila melanogaster; C.e., Caenorhabditis elegans; S.c., Saccharomyces cerevisiae; S.p., Schizosaccharomyces pombe. No similarity is found between human domain $4 \mathrm{~b}$ and $5 \mathrm{~b}$ sequences and protein sequences from non-vertebrate databases. (c) Probability of coiled-coil structure for domain $5 \mathrm{~b}$ in man and Fugu 
sequences indicated that domain 4, corresponding to Ex4, shares broad homologies among vertebrates, but restricted similarities with invertebrate and yeast sequences (Figure $1 b$ ). Domains $4 b$ and $5 b$, corresponding to Ex4b and Ex5b, are found only in vertebrate genomes, exemplified here in mammal and fish sequences (Figure 1b), and are absent from Drosophila, Caenorhabditis and lower eucaryote genomic sequences. The 17 amino acids of domain $4 \mathrm{~b}$ are well conserved but do not display any specific motif or structure. The first 24 residues of the 37-amino-acid long domain $5 \mathrm{~b}$ are highly conserved and the whole domain displays a highly conserved coiled-coil structure predicted to homopolymerize (Figure 1c). To gain insight into the identification of OPA1 variant expression, we performed quantitative polymerase chain reaction (Q-PCR) on cDNA from HeLa cells, using couples of oligonucleotides specific of each of the eight mRNA variants. The OPA1-Ex4 mRNA variant \#1 was arbitrarily chosen as reference, as it was the first OPA1 sequence identified (AB011139), ${ }^{28}$ and since then selected in most reported OPA1 ectopic expression experiments. Our Q-PCR data suggest that four OPA1 mRNA variants predominate: Ex4, Ex4+Ex4b, Ex4+Ex5b and Ex4 $+E x 4 b+E x 5 b$, the other four being 25-40 times less abundant than OPA1-Ex4 mRNA (Figure 1a and Supplementary Table 1). To correlate these mRNA abundances with the five bands observed by Western blots in HeLa cells (denoted 'a' to 'e'), we have taken into account data from OPA1 isoform overexpression (Figure 2A) and silencing (Figure 4A) experiments, and data reported by Ishihara et al., ${ }^{21}$ showing that OPA1 isoforms are present as a large (L) and one or two short (S) forms, resulting from proteolytic cleavages in domains 5 and $5 \mathrm{~b}$, when present. In our experiments, we have first overexpressed OPA1 isoforms containing either none (OPA1-0) or one of three domains (OPA1-4, OPA1-4b and OPA1-5b), using the corresponding open reading frames including Ex4, or Ex4b or Ex5b or none, and then knocked down OPA1 mRNAs using Ex4-, Ex4b- and Ex5b-specific siRNAs. Results from overexpression experiments suggest that OPA1-0, OPA-4 and OPA-5b generate L-forms migrating at the level of band 'b', although a little bit lower for OPA1-0, and S-forms migrating as band 'e' for OPA1-0 and OPA1-4 and band ' $d$ ' and ' $c$ ' for OPA1-5b, as expected and reported. ${ }^{21}$ Nevertheless, OPA1-4b overexpression led to a single Sform migrating at the level of band ' $d$ ', suggesting that the OPA1-4b L-form is completely processed not in domain 5 as expected, but probably in domain $4 \mathrm{~b}$ to generate a peptide, which length is compatible with the migration of band ' $d$ ' (Supplementary Table 2). Assuming this eventual new cleavage site in domain $4 \mathrm{~b}$ that would prevent the cleavage in domain 5, one can readily analyse the Western blot obtained for the silencing experiment described in Figure 4A (see Supplementary Table 2). Together, these data suggest that bands ' $a$ ' and 'b' correspond to L-forms and bands 'c', ' $d$ ' and ' $e$ ' to S-forms resulting from different proteolytic cleavages, which we compiled on Figure 1a.

Phenotype associated to OPA1 variant expression. To address the function of domains $4,4 \mathrm{~b}$ and $5 \mathrm{~b}$, we compared the overexpression effects of the OPA1-0, OPA1-4, OPA1- 4b and OPA1-5b isoforms in fission yeast and HeLa cells. In the Schizosaccharomyces pombe strain deleted for msp1 gene, OPA1-0, OPA1-4 and OPA1-4b expression rescues the absence of the Msp1 protein (Figure 2A), whereas this was not true when OPA1 ectopic expression is repressed (not shown) or when OPA1-5b is expressed, suggesting that independently of the presence of Ex4 or Ex4b, OPA1 can replace the Msp1 mitochondrial functions, but not when Ex5b is included in OPA1 open reading frame.

We then transfected in HeLa cells vectors expressing each variant and detected the ectopic OPA1 by Western blot and indirect immunofluorescence (IF). Twenty-four hours after transfection, mitochondrial phenotypes were observed using Mitotracker Red staining and IF using OPA1 antibody dilution that barely detects endogenous OPA1 expression. Around $35 \%$ of the cells were strongly stained by IF and showed fragmented mitochondrial networks colocalizing with the Mitotracker fluorescence (Figure $2 \mathrm{C}(\mathrm{a}-\mathrm{h})$ ). Overexpression of OPA1-0, OPA1-4 and OPA1-4b led to intermediate fragmented figures and punctuated mitochondria in almost equal proportions (Figure $2 \mathrm{~B}$ and $\mathrm{C}(\mathrm{a}-\mathrm{f})$ ). Cells with a low exogenous OPA1 level had mitochondria appearing as short tubules, whereas cells with high overexpression level had mitochondria appearing as dots (not shown). In addition, expression of OPA1-5b led to a homogeneous phenotype with extremely small vesicular mitochondria (Figure $2 \mathrm{~B}$ and $\mathrm{C}(\mathrm{g}$, h)), contrasting with the phenotype in surrounding untransfected cells (Figure 2C, stars), or in YFP-transfected cells (Figure 2B(i, j)). To address the possible relationship between the mitochondrial network fission and a $\Delta \Psi_{\mathrm{m}}$ dissipation, a qualitative analysis was performed using the $\mathrm{JC}-1$ probe. Although no $\Delta \Psi_{\mathrm{m}}$ dissipation was observed in control cells, expression of OPA 1 isoforms 0,4 or $4 \mathrm{~b}$ produced around $15 \%$ of cells totally devoid of red $\mathrm{JC}-1$ aggregates. This value reached $30 \%$ in cultures expressing the OPA $1-5 \mathrm{~b}$ isoform. As all cells with $\Delta \Psi_{\mathrm{m}}$ dissipation displayed a punctuate mitochondrial network and overexpression of OPA1 variants induced fragmentation of the mitochondrial network, we assume that the cells with $\Delta \Psi_{\mathrm{m}}$ dissipation correspond to transfected cells and consequently that mitochondrial fragmentation induced by OPA1 isoform overexpression correlates with progressive $\Delta \Psi_{\mathrm{m}}$ dissipation (see Supplementary figure). We further examined the effect of the overexpression of OPA1 isoforms on the mitochondrial ultrastructure by TEM. HeLa cells were microinjected with YFP or OPA1 variant expressing vectors, together with immunoglobulin conjugated to $10 \mathrm{~nm}$ gold beads to locate injected cells. Cells overexpressing OPA1 had predominantly small spherical mitochondria (Figure 2D(a-d)), compared to the filamentous shape of mitochondria in control cells (Figure 2D(e)). Nevertheless, although mitochondria of control cells or cells expressing OPA1-0, OPA1-4 or OPA1-4b showed normal cristae structure with a regular shape (Figure $2 \mathrm{D}(\mathrm{a}-\mathrm{c}, \mathrm{e}))$, the cristae structure of cells expressing OPA $1-5 \mathrm{~b}$ were dramatically modified, reminiscent of apoptotic mitochondria (Figure 2D(d)). To assess possible apoptotic events, we used a direct DAPI-labelling procedure that prevents cells detaching from the support. In HeLa cells transfected by OPA1-0, OPA1-4 and OPA1-4b or YFP, similar background levels of apoptotic-like nuclei, with condensed and fragmented chromatin, were observed. In contrast, $16 \%$ of cells 
A
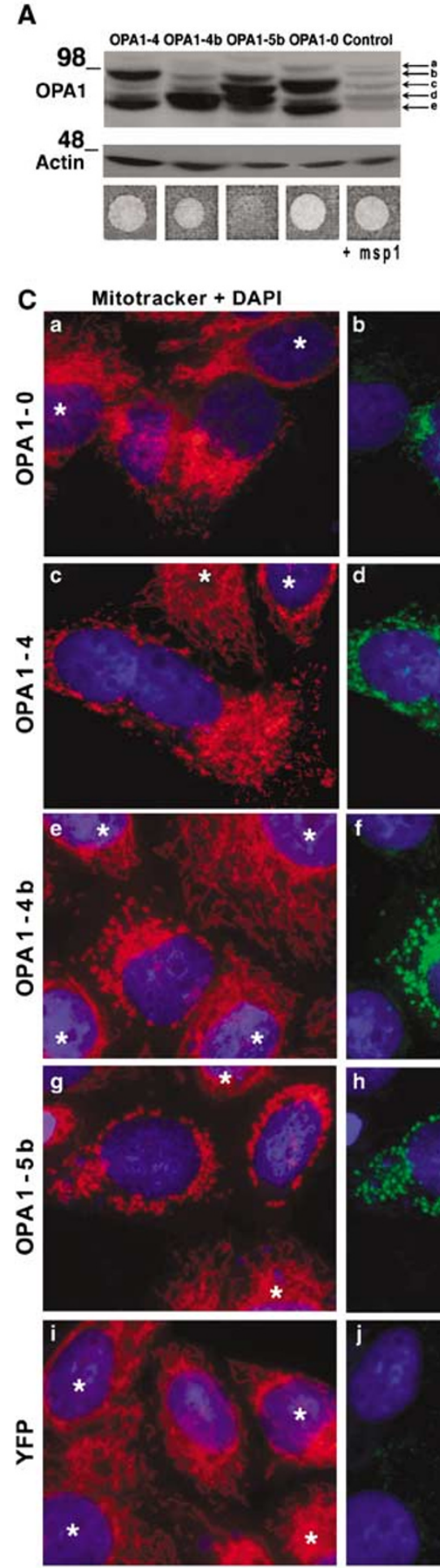

B

\begin{tabular}{|c|c|c|c|}
\hline & filamentous & fragmentated & punetuated \\
\hline OPA1-0 & $3 \%$ & $56 \%$ & $41 \%$ \\
\hline OPA1-4 & $1.3 \%$ & $43.6 \%$ & $55 \%$ \\
\hline OPA1-4b & $0 \%$ & $41 \%$ & $59 \%$ \\
\hline OPA1-5b & $0 \%$ & $8 \%$ & $92 \%$ \\
\hline YFP & $96 \%$ & $4 \%$ & $0 \%$ \\
\hline
\end{tabular}

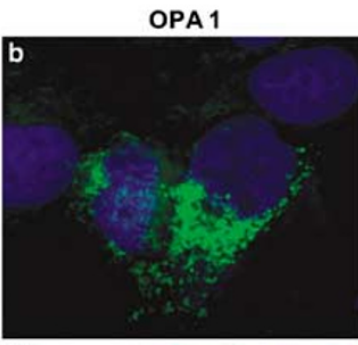

D
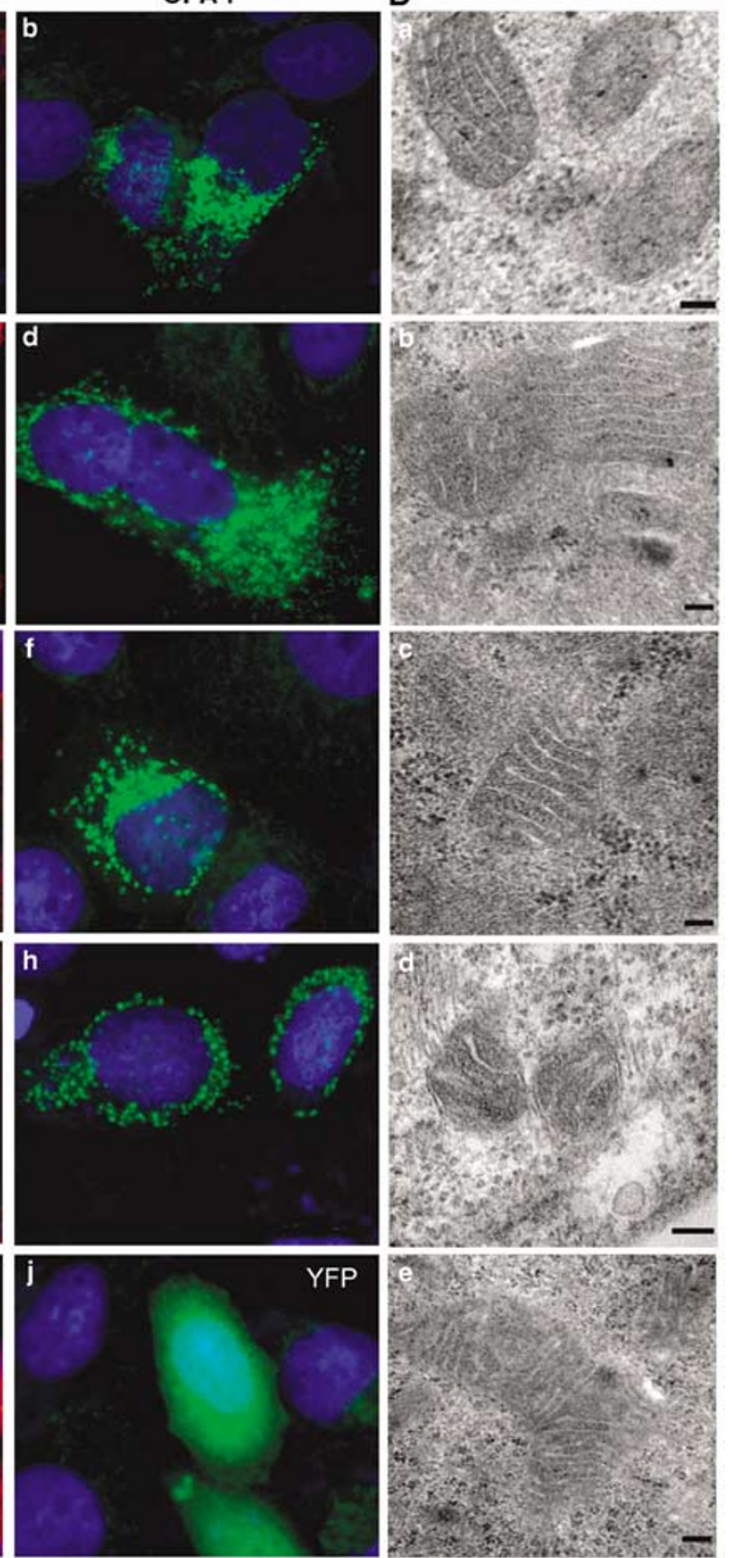

Figure 2 Fission yeast complementation test and OPA1 variant expression phenotypes in HeLa cells. Complementation tests were performed in a diploid fission yeast strain deleted for msp1, transfected with plasmid expressing OPA1-0, OPA1-4, OPA1-4b, OPA1-5b or msp1. HeLa cells were transfected with plasmids expressing OPA1-0, OPA1-4, OPA1-4b, OPA1-5b, or control plasmids expressing YFP and processed $24 \mathrm{~h}$ after transfection. (A) Top: Western blot analyses of whole HeLa cell extracts probed with anti-OPA1 (1/2000) or anti-actin (1/10000) antibodies. Endogenous OPA1 signal is shown in mock-transfected cells. Actin labelling was used as a loading control. Bottom: 5000 spores originating from the diploid strain deleted for msp1 were spotted on a medium lacking thiamine to induce OPA1 variant expression; the positive control $(+m s p 1)$ on the right corresponds to the use of the pREP41-msp1 plasmid. (B) Percentage of phenotypes shown above the table, among transfected HeLa cells. (C) Mitotracker Red and DAPI staining ( $a, c, e, g$, i) and IF anti-OPA1 labelling (b, $d, f, h)$ or YFP (j) fluorescence in transfected cells. Untransfected cells are shown by stars. (D) TEM micrographs of thin section of HeLa cells showing the ultrastructure of mitochondria $24 \mathrm{~h}$ after microinjection of plasmids. Bars $100 \mathrm{~nm}$ 
transfected by OPA-5b had a phenotype reminiscent of apoptosis (Figure 3A). In all cases, treatment with the caspase inhibitor z-VAD-fmk significantly reduced the amount of apoptotic cells, suggesting that the previous apoptotic process involved activation of caspase cascades. The apoptotic nuclear phenotype correlated with cyt $c$ release in the cytoplasm in cells overexpressing OPA1-5b (9\%) (Figure $3 \mathrm{~B}(\mathrm{j}-\mathrm{l}))$, whereas no cyt $c$ release was observed in cells transfected with the other variants (Figure $3 \mathrm{~B}(\mathrm{a}-\mathrm{i})$ ) or in control cells. Thus, independently of the presence of the alternate domains, overexpression of the different OPA1 variants induced a membrane potential dissipation and
A

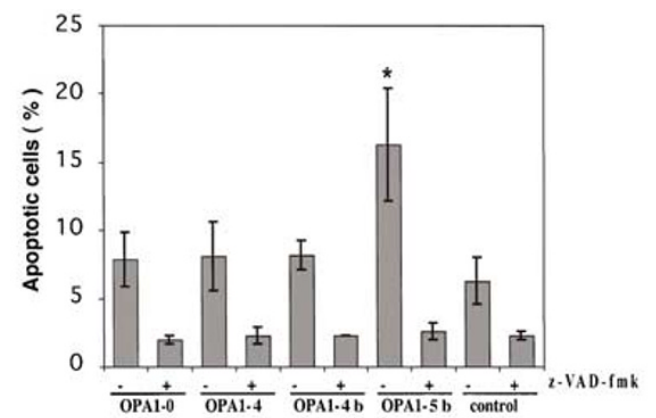

B
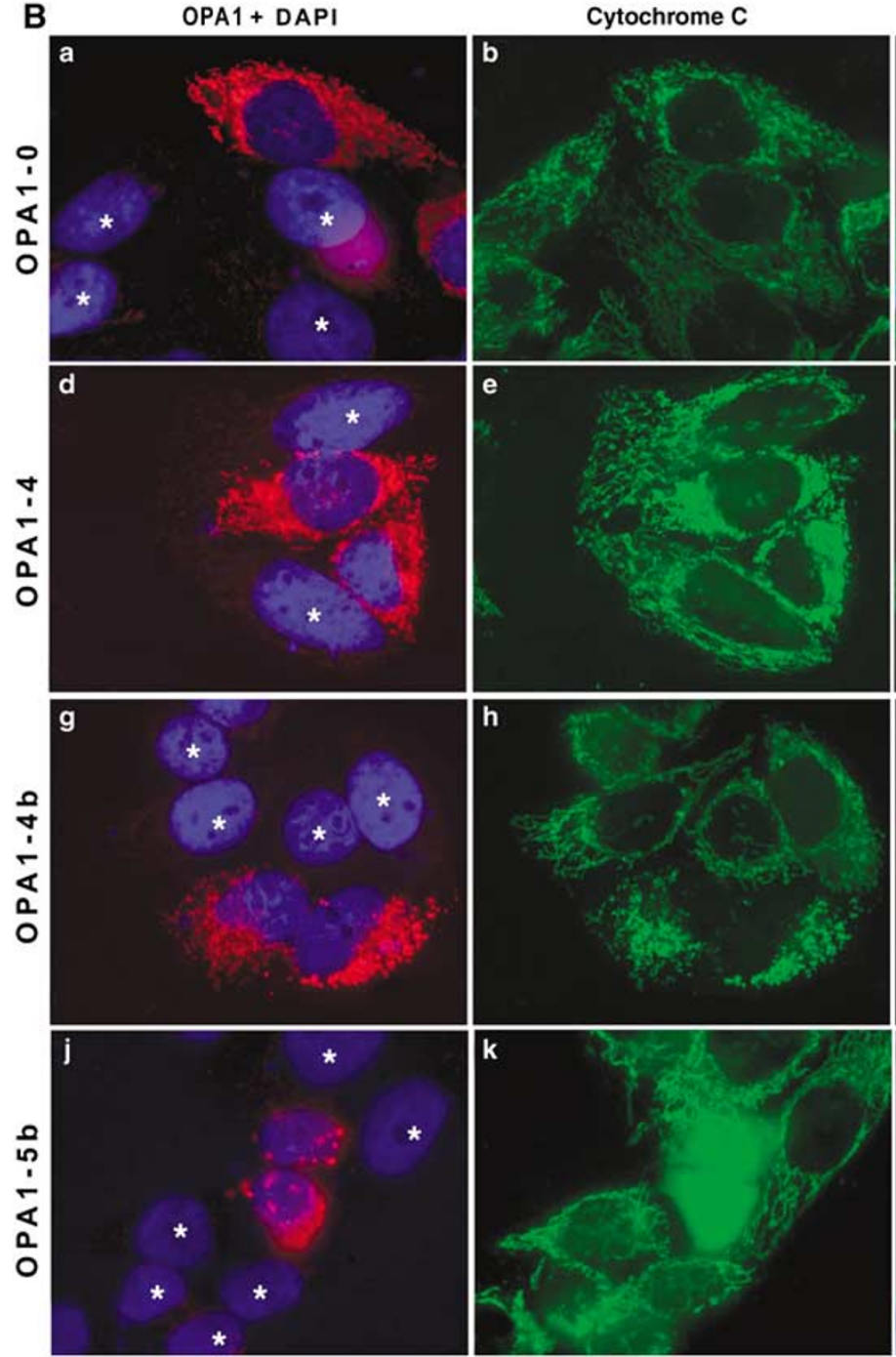

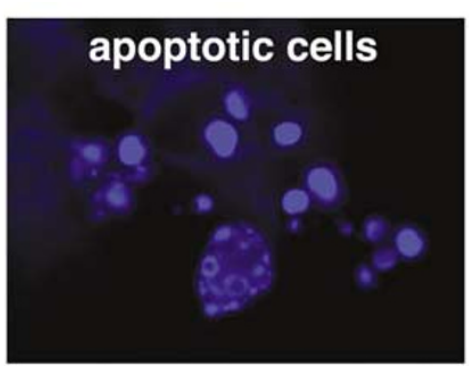

Merge
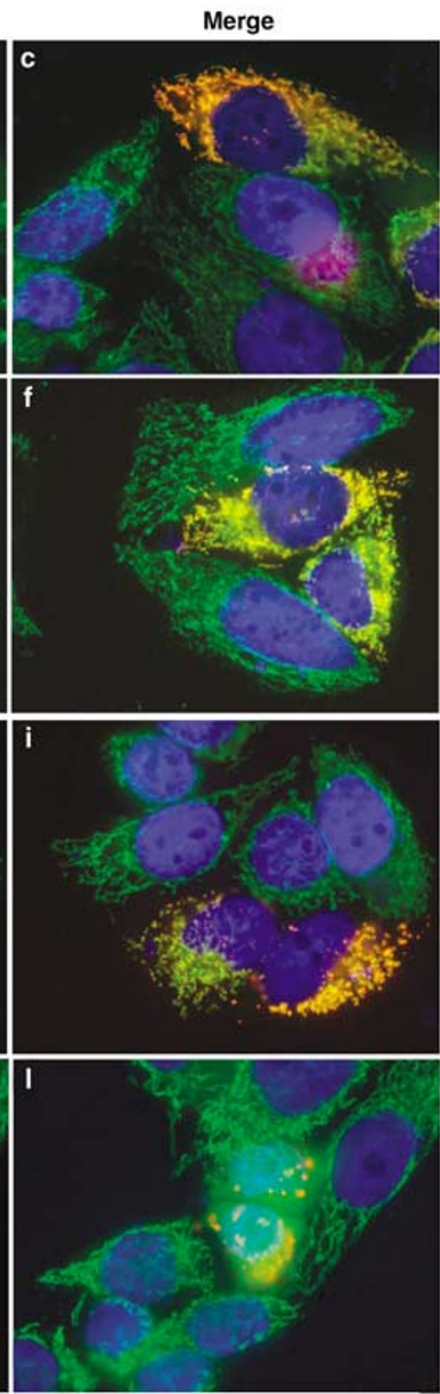

Figure 3 Cyt $c$ release correlates with OPA1-5b-induced apoptosis. HeLa cells were transfected with plasmids expressing OPA1-0, OPA1-4, OPA1-4b, OPA1-5b and processed $24 \mathrm{~h}$ after transfection. (A) Quantification of apoptotic nuclei with DAPI staining (as shown on the right) in the presence or absence of $\mathrm{z}$-VAD-fmk (100 $\mu \mathrm{M}$ ). Black star denotes a significant increased value comparatively to control cells $(P<0.05)$. (B) Cells, treated with z-VAD-fmk $(100 \mu \mathrm{M}) 4 \mathrm{~h}$ after transfection, were fixed at $24 \mathrm{~h}$, stained with DAPI and immuno-labelled with anti-OPA1 (1/800) (red: a, d, g, j) and anti-cyt c (green: b, e, h, k). Merged (c, f, i, I). Untransfected cells are shown by white stars 
A

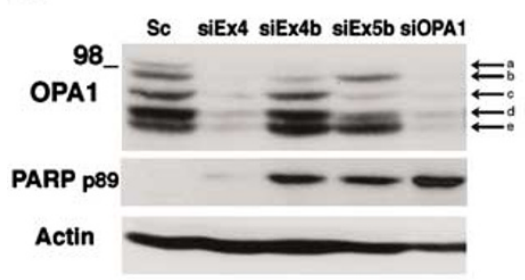

B
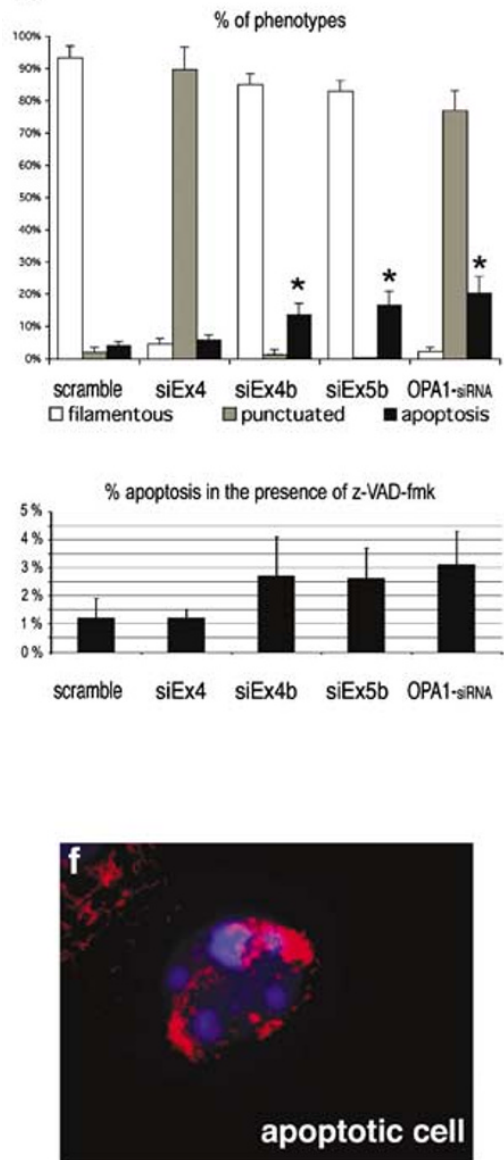

C

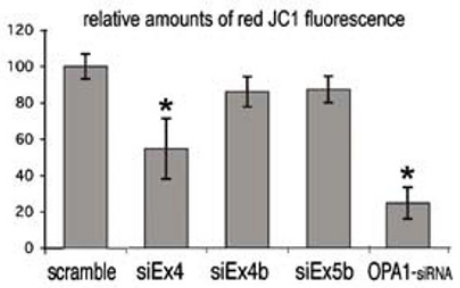

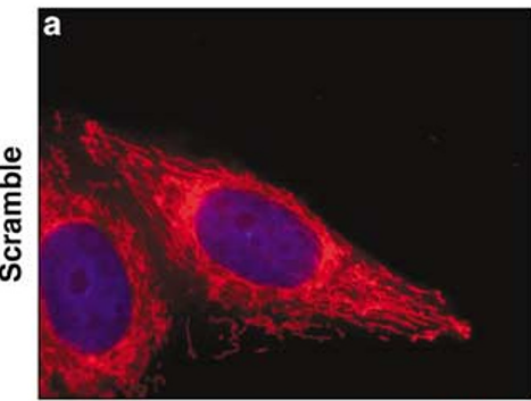
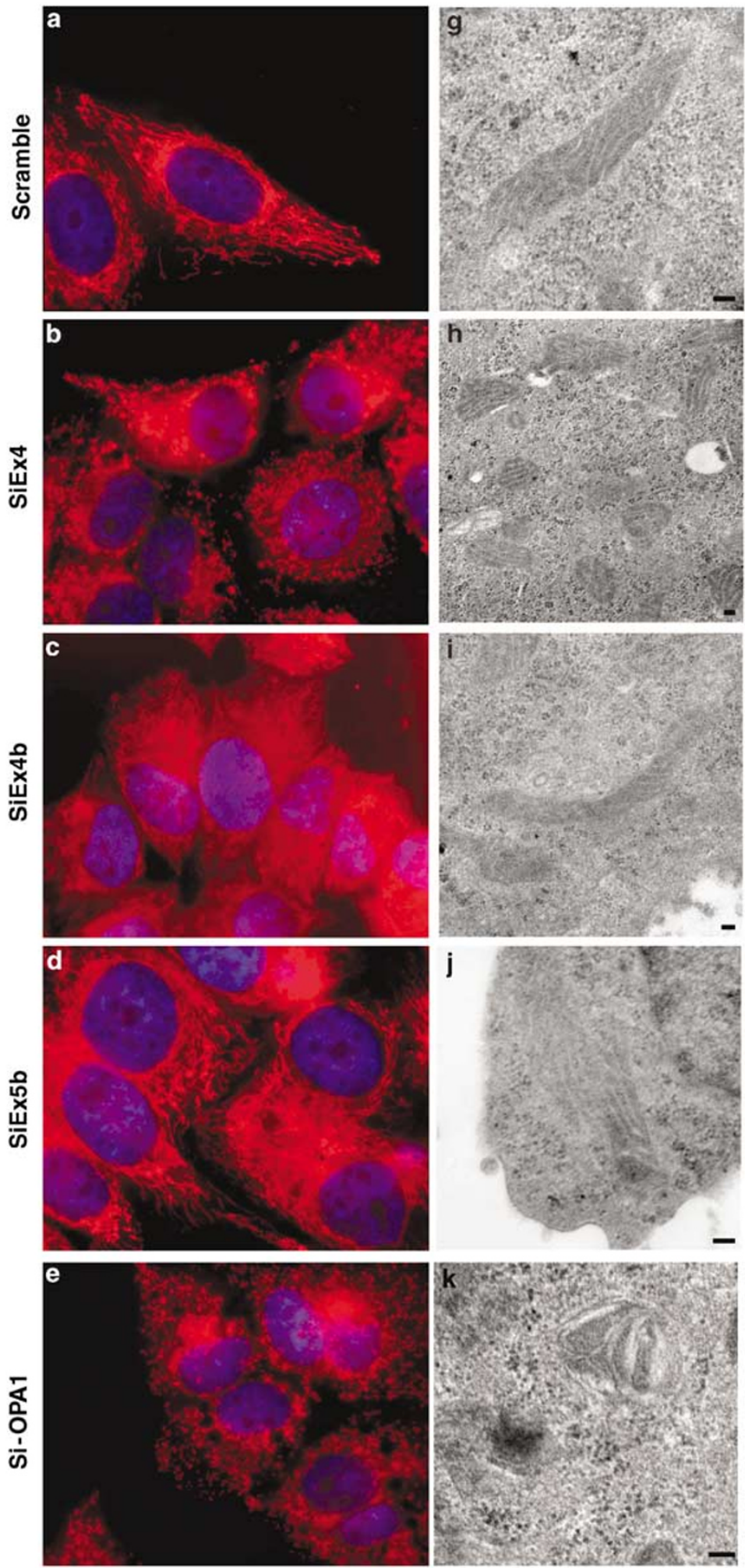

Figure 4 Exon-specific siRNA uncouples functions in mitochondrial network fusion and apoptosis. HeLa cells were transfected with siRNA matching the alternatively spliced exons, siEx4, siEx4b and siEx5b, with the OPA1-siRNA, or with the scramble siRNA as control, and processed $72 \mathrm{~h}$ after transfection. (A) Western blot analyses of whole-cell extracts, probed with anti-OPA1 (1/400) or anti-actin (1/10000) antibodies. (B) Analysis of Mitotracker Red and DAPI staining by fluorescence microscopy (a-f). Top graph: percentage of cells showing a filamentous or punctuate mitochondrial network, or showing an apoptotic phenotype (as in f). Bottom graph: percentage of apoptosis in the presence of z-VAD-fmk. Transmission electron microscope micrographs of thin section of HeLa cells showing the ultrastructure of mitochondria ( $\mathrm{g}-\mathrm{k})$. Bars: $100 \mathrm{~nm}$. (C) $\Delta \Psi_{\mathrm{m}}$ assessment using JC-1 dye. Asterisks in histograms denote significantly increased (in $\mathrm{B}$ ) or decreased (in $\mathrm{C}$ ) values comparatively to control cells $(P<0.05)$ 
fragmentation of the mitochondrial network. In addition, the overexpression of OPA1-5b induced an apoptotic process with major cristae remodelling and $c y t c$ release, suggesting that domain $5 b$ interacts with the apoptotic process.

\section{Phenotype associated to OPA1 alternate splicing exon} silencing. To further investigate whether specific functions are associated with OPA1 domains, we selectively inhibited the expression of the endogenous OPA1 variants. Three siRNAs: siEx4, siEx4b and siEx5b were transfected into HeLa cells, and their effects studied $72 \mathrm{~h}$ later in comparison with an siRNA targeting all OPA1 variants (OPA1-siRNA) and a scramble siRNA. ${ }^{11}$ Silencing of OPA1 variants that contain the Ex4 led to the disappearance of the four most abundant OPA1 isoforms, while silencing of variants containing the Ex4b and Ex5b led to a pattern compatible with the target sequences (Figure $4 \mathrm{C}$ and Supplementary Table 2). Examination of mitochondrial network morphology with Mitotracker Red showed that control cells had a filamentous mitochondrial network, whereas virtually all cells transfected with siEx4 or OPA1-siRNA showed a punctuated mitochondrial network (Figure 4B(b, e)). Conversely, cells transfected with either siEx4b or siEx5b showed a highly filamentous mitochondrial network (Figure 4B(c, d)), which appeared even thinner and more interconnected than in control cells (Figure $4 \mathrm{~B}(\mathrm{a})) . \Delta \Psi_{\mathrm{m}}$ measurement was performed by a spectroscopic quantitative approach using the $\mathrm{JC}-1$ probe (Figure $4 \mathrm{C}$ ) assessing the red to green fluorescence ratio. Significant $\Delta \Psi_{\mathrm{m}}$ dissipation was observed in cells transfected with the siEx4 and was more extensive in cells transfected with OPA1-siRNA, consistent with the fragmented mitochondrial phenotype. In cells transfected with siEx $4 \mathrm{~b}$ and siEx5b, only a slight decrease of the $\Delta \Psi_{\mathrm{m}}$ was found, compared to cells transfected with the scramble siRNA. Labelling chromatin with DAPI revealed that a significant proportion (Figure 4B, top graph) of the cells transfected with siEx4b, siEx5b or OPA1-siRNA showed condensed and fragmented chromatin, a phenotype reminiscent of apoptotic cells (Figure $4 B(f)$ ), which was absent from cells transfected with siEx4 or the scramble siRNA. To confirm that downregulation of OPA1 variants containing Ex4b or Ex5b induced apoptosis, we assessed by Western blot the PARP cleavage that indeed correlates with the apoptotic phenotype (Figure 4A). In addition, concomitant treatment with $z-V A D-f m k$ induced a global $80 \%$ reduction of apoptosis in cultures treated with siEx4b, siEx5b and OPA1siRNA, suggesting that the apoptotic process involve caspase cascades (Figure 4B, bottom graph). To further study, the apoptotic process, we monitored in transfected cells the status of the cyt $c$, using specific antibodies, and the Mitotracker dye (Figure 5B). In control and siEx4-transfected cells, cyt $c$ IF perfectly colocalized with the Mitotracker fluorescence in mitochondria. In siEx4b- and siEx5btransfected cultures, some cells $(6 \%)$ showed partial release of cyt $c$ (phenotype a) with the mitochondrial network still highly tubular. Very few cells $(<0.5 \%)$ showed a complete release of $c y t c$ and Mitotracker fluorescence into the mitochondria (phenotype b). In addition, we evidenced cells $(>2 \%$ ) with a uniform cytoplasmic cyt $c$ fluorescence and an unusual uniform cytoplasmic Mitotracker fluorescence (phenotype c), suggesting that in these cells the membrane potential was beyond the minimal level required for the Mitotracker accumulation into mitochondria. In cells treated with the OPA1-siRNA, a significant proportion showed a partial $(7 \%)$ or complete $(22 \%)$ release of cyt $c$, with always a uniform Mitotracker fluorescence in the mitochondrial network. Finally, we explored the mitochondrial ultrastructures by TEM (Figure $4 \mathrm{~B}(\mathrm{~g}-\mathrm{k}))$. Consistent with former observations, mitochondrial shapes were tubular for control cells and siEx4b- and siEx5b-transfected cells (Figure $4 \mathrm{~B}(\mathrm{~g}, \mathrm{i}, \mathrm{j})$ ), and spherical in siEx4- and OPA1-siRNA-transfected cells (Figure 4B(h, k)). Surprisingly, no major perturbations of the IMM structure, and in particular of the cristae morphology, were observed in cells transfected by the exon-specific siRNAs or in control cells (Figure $4 \mathrm{~B}(\mathrm{~g}-\mathrm{j})$ ), in contrast to cells transfected by the OPA1-siRNA (Figure 4B(k)).

\begin{abstract}
Abundance of OPA1 mRNA variants in different human organs. Although mitochondria from different cell types and organs present sometimes highly different membrane structures and consequently bioenergetic capacities, our knowledge on the actors that define the IMM and OMM shapes remains evasive. To provide insights into this question, we have monitored by Q-PCR the relative abundances of OPA1 variants in cDNAs from different organ (Figure 6 and Supplementary Table 1). Our data, calculated in reference to OPA1-Ex4 variant, show great variability according to the organ, although Ex4-containing mRNAs are systematically abundant. Nevertheless, in the liver, kidney and thymus, the presence of Ex5b predominates among all variants, whereas in the brain, Ex4b is highly expressed and Ex5b barely detected. Finally in the heart, Ex4 predominates, with under representation of Ex4b and Ex5b. Together, these results suggest that the mitochondrial fate might be regulated by selective alternate splicing of OPA1 mRNA.
\end{abstract}

\section{Discussion}

IMM dynamic regulation is of crucial interest, as this membrane participates both in the global mitochondrial network dynamic, probably by indirect interactions with the OMM, and to more intimate mechanisms associated to the cellular bioenergetic status and the apoptotic process. To date, few actors of the IMM dynamic have been identified in mammal cells, among them the MTP18 protein, ${ }^{29}$ the mitofilin $^{30}$ and OPA1. The fact that the OPA1 protein exists as eight different isoforms in vertebrates suggests that these proteins can fulfil different functions inside mitochondria.

OPA1 involvement in mitochondrial network dynamic and $\Delta \Psi_{m}$ maintenance. Our results from OPA1 variant expression experiments showed that, whatever the isoform, OPA1 overexpression in cells with tubular mitochondria as HeLa cells induced fission of the mitochondrial network. This was already observed by others, ${ }^{15}$ and suggests that drastic increase of OPA1 abundance interfere with endogenous OPA1 and has an indirect negative effect on the ability to fuse the mitochondrial network. Indeed, in cells with 

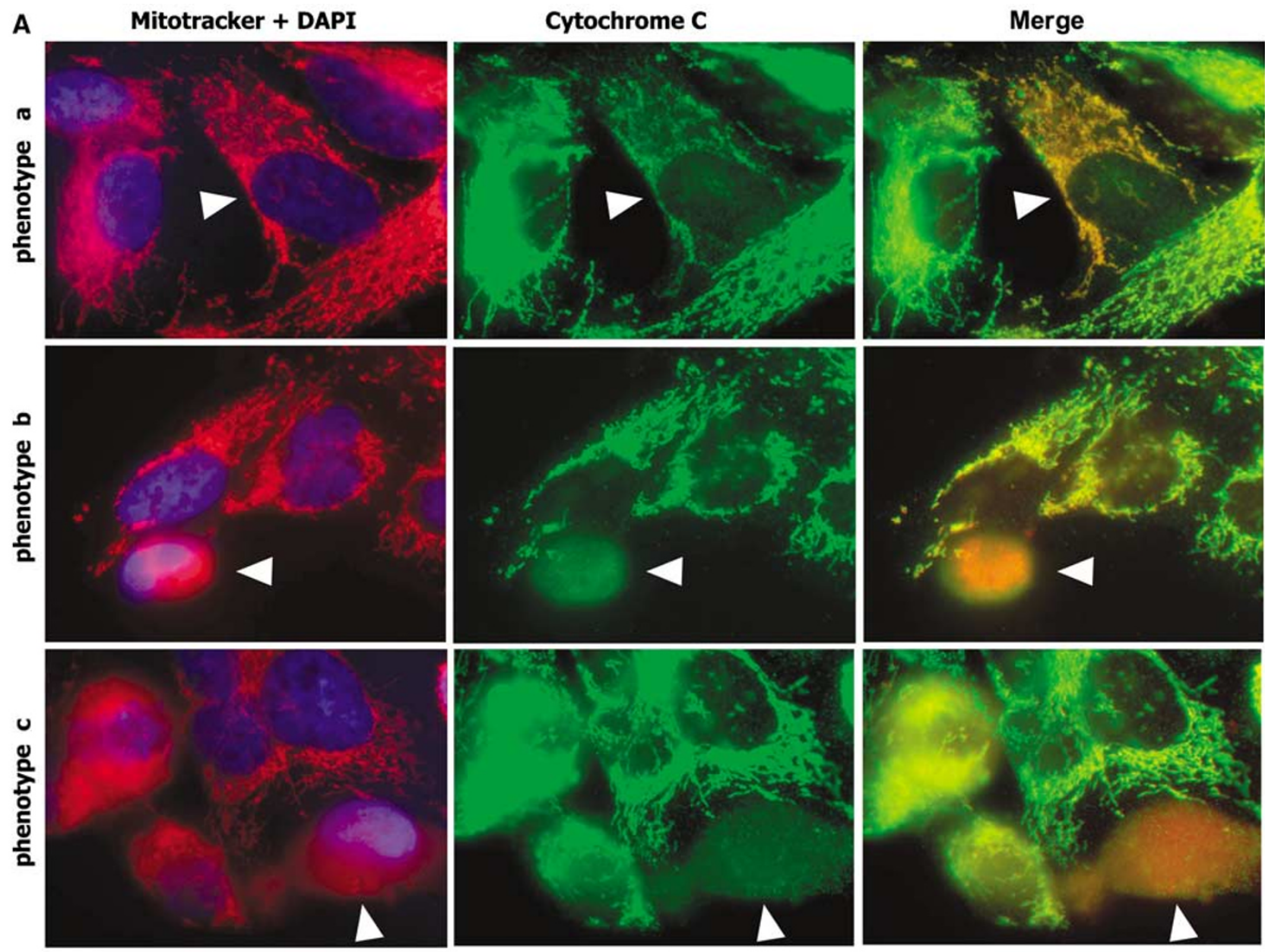

B

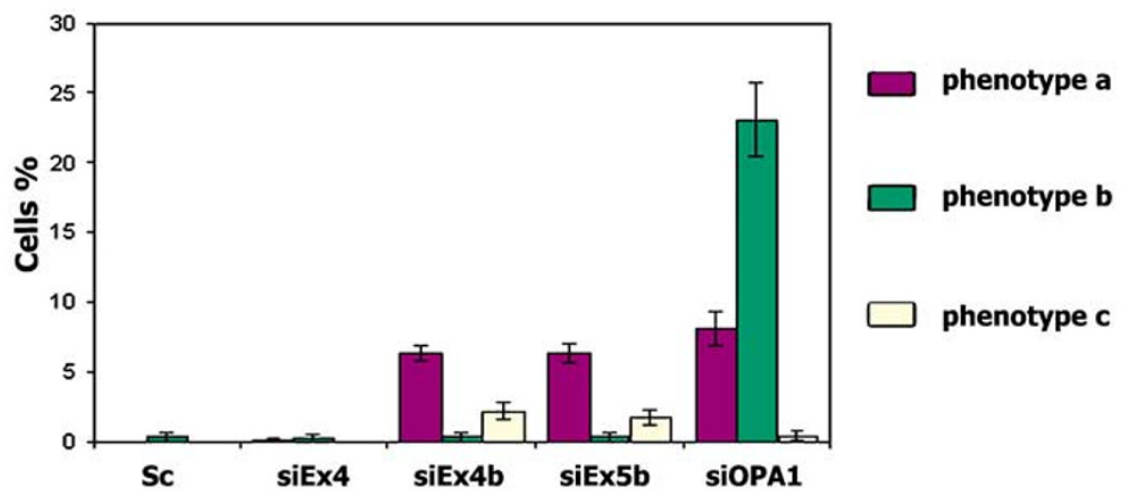

Figure 5 Assessment of $c y t c$ release in cells transfected with the different siRNAs. HeLa cells were transfected by the five different siRNA, then processed after $72 \mathrm{~h}$ for cyt $c$ IF and Mitotracker Red fluorescence and DAPI staining. (A): Phenotypes observed: (a) cyt c partially released in the cytoplasm; (b) cyt $c$ completely released in the cytoplasm and mitochondrial Mitotracker labelling; (c) cyt c completely released in the cytoplasm and cytoplasmic Mitotracker florescence. (B) Relative amounts of the three phenotypes in the siRNA-transfected cultures

physiologically punctuated mitochondria, as Cos7, increasing OPA1 expression induces the fusion of the mitochondrial network. $^{7}$ In addition, we and others have shown that the global disruption of OPA1 expression by siRNA blocks mitochondrial fusion. ${ }^{11,15-18}$ In this work, we reported that the silencing of OPA1 variants containing Ex4, which encode the most abundant isoforms in HeLa cells, induces a similar mitochondrial fragmentation and $\Delta \Psi_{\mathrm{m}}$ dissipation, without consequence on cyt $c$ release and apoptosis. This suggests that Ex4-containing variants are required to maintain the network in a fusion state. Because no modification of the IMM structure was detected by conventional TEM, one can speculate that the silencing of Ex4-containing OPA1 isoforms affects the IMM integrity and bioenergetic status that would 

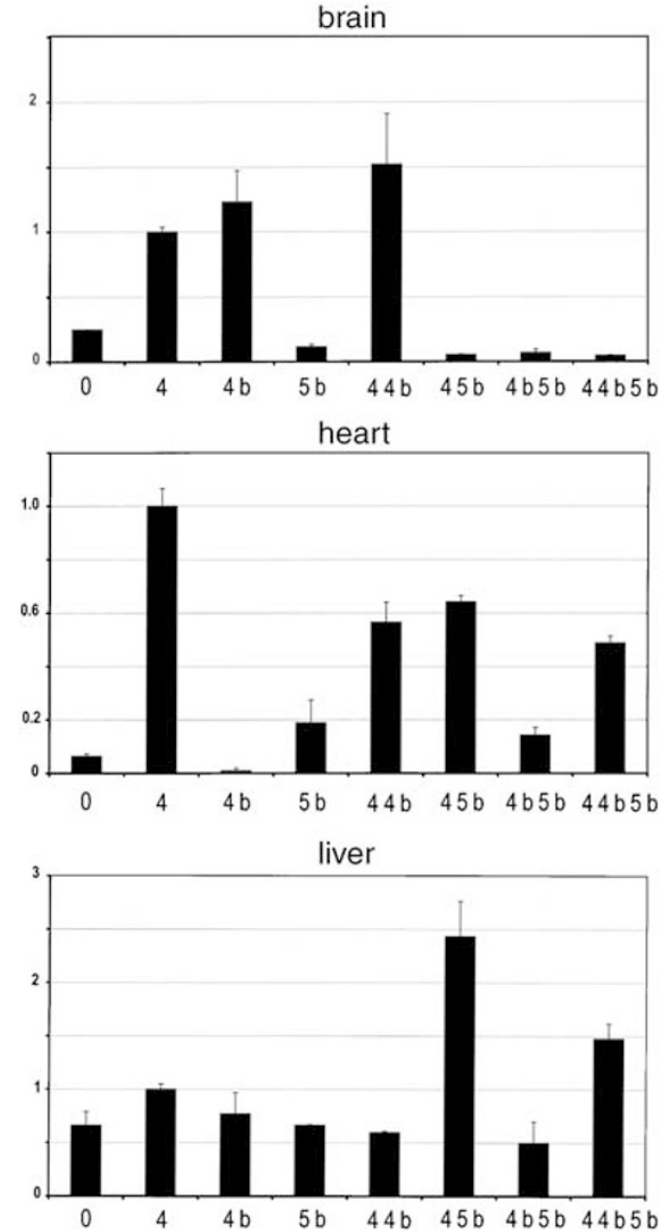

kidney
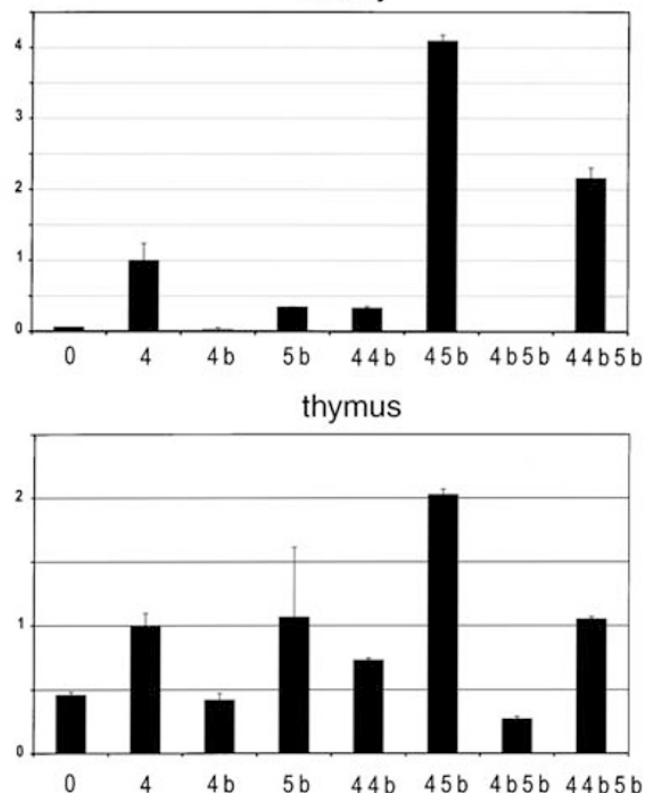

Figure 6 Relative abundance of OPA1 mRNA variants in human tissues. cDNA were retro-transcribed on total RNA from human brain, heart, liver, kidney and thymus. Q-PCRs were performed on identical amounts of CDNA, using couples of oligonucleotides specific of each OPA1 variant. OPA1-Ex4 mRNA abundance was arbitrarily set to 1 and used to calculated the other isoform abundances consequently induce the fission of the mitochondrial network. ${ }^{10}$ Alternatively, domain 4 might be required to interact with the Mfn1/Mfn2 protein to promote mitochondrial fusion. ${ }^{12}$ In both cases, the presence of domain 4 in OPA1 specifies functions associated with the $\Delta \Psi_{\mathrm{m}}$ maintenance and fusion of the mitochondrial network, functions that have been associated to OPA1 orthologues throughout evolution. ${ }^{24,25,31}$ Conversely, results from Ex4b and Ex5b silencing experiments exclude their involvement in the fusion process or in the membrane potential maintenance.

OPA1 involvement in the apoptotic process. Our results from OPA1-5b overexpression and Ex4b and Ex5b silencing evidenced two types of apoptotic processes based on cyt $c$ release and caspase cascades. The first one, already described for the silencing of all OPA1 isoforms, is similar to that found for overexpression of OPA1-5b and corresponds to most apoptotic processes described in the literature. ${ }^{1,2}$ Cells undergo mitochondrial fission, $\Delta \Psi_{\mathrm{m}}$ dissipation, cytoplasm shrinkage, major modifications of the cristae structure and rapid and complete cyt $c$ release to terminate with nuclear fragmentation. Our results from OPA1-5b overexpression suggest that domain $5 b$ has a specific function or conformation that might affect the sequestration of cyt $c$ in vertebrate cells and confer to OPA1 a property incompatible with the rescue of msp1 deletion in fission yeast. The second form of apoptosis was observed when OPA1 variants containing Ex4b or Ex5b were silenced. In these experiments, cells undergo apoptosis without mitochondrial network fission, without major $\Delta \Psi_{\mathrm{m}}$ dissipation, with very few cells showing complete cyt $c$ release and without major modifications of the cristae structure. These observations suggest that discrete modifications of the IMM, undetectable by conventional TEM, are sufficient for a progressive, probably slow process of cyt $c$ mobilization and release, which do not affect immediately other processes associated with IMM integrity. The fact that $c y t c$ release occurs as a slow process when mitochondria are tubular might illustrate why in most apoptotic process extensive cyt $c$ release is concomitant to the mitochondrial network fission. In this respect, our observations converge with the fact that apoptotis can be strongly inhibited when OMM fission is suppressed by the downregulation or mutation of fission actors, as Drp1 and hFis1. ${ }^{6,32}$ Slow cyt $c$ release has also been observed in neurones treated with low doses of rotenone, a powerful respiratory complex 1 inhibitor, suggesting that cyt $c$ release is not always an 'all-or-none' process ${ }^{33}$ and that tight regulation of cyt $c$ sequestration can be achieved by mild modifications of OPA1. The maintenance of a tubular network in siEx4b and siEx5b correlates well with the maintenance of the $\Delta \Psi_{\mathrm{m}}$, although in some cells we observed a complete dissipation of $\Delta \Psi_{\mathrm{m}}$ that might be correlated to the ultimate lack of $c y t c$ in the mitochondria and consequently respiration impairment. As the absence of domain $4 \mathrm{~b}$ or $5 \mathrm{~b}$ led to this apoptotic process, one might speculate that OPA1 isoforms including these domains have a specific and restricted function in trapping cyt $c$ inside the mitochondria and in particular within the cristae. Because cristae junction remodelling, cyt $c$ release during 
apoptosis $^{3,9,13}$ and alternate splicing in OPA1 have only been described in vertebrates, we propose that these processes result from co-evolution and constitute a vertebrate-specific mechanism controlling segregation of cyt $c$. Domain $5 \mathrm{~b}$ that is predicted to form a homopolymerizing coiled-coil structure might induce a higher level of OPA1 assembly compatible with the tight structure 'plugging' cristae junction. Its absence would mobilize cyt $c$ into the IMS and allow a subsequent slow release into the cytoplasm, without initial modification of other mitochondrial functions. Similarly, domain $4 \mathrm{~b}$ could positively regulate this complex structure and we can hypothesize that it might be the target of proapoptotic proteins, as tBid or Bik,, ${ }^{3,9}$ to further dismantle domain $5 \mathrm{~b}$ coiled-coil polymers and mobilize cyt $c$ in the IMS.

Uncoupling mitochondrial fusion/fission process from apoptosis. Results from OPA1 mRNA-specific silencing experiments have shown that by acting on different OPA1 isoforms, one can induce mitochondrial fission without inducing apoptosis, and conversely, induce apoptosis without mitochondrial fission. These observations are consistent with the existence of two different pathways regulating the different OPA1 isoforms and supported by the recent identification of the PARL and Paraplegin proteases, respectively, as OPA1-specific processors involved in and exclusively apoptosis and mitochondrial morphology. ${ }^{21,22} \mathrm{We}$ can speculate from these and our works that the PARL cleavage might target OPA1 domains involved in specifying apoptotic functions (domains $4 \mathrm{~b}$ and $5 \mathrm{~b}$ ) and that the Paraplegin cleavage might affect an OPA1 evolutionary conserved domain, which is proposed to be domain $5 .^{21}$ Future experiments that will uncover the proteins interacting with OPA1 amino-terminus region will shed light on these hypothesis.

Mitochondrial fate associated to OPA1 alternate splicing. Finally, our observations that OPA1 variants greatly vary between organs suggest that mitochondrial fusion and susceptibility to apoptosis might be basically regulated by the nuclear splicing machinery, and consequently that the relative abundance of each OPA1 isoform can contribute to adapt mitochondrial dynamic to cell types and growth conditions. In this respect, characterization of the expression levels of each variant in human retinal ganglion cells might contribute to identify the pathophysiological mechanism responsible for their susceptibility to degeneration in OPA1-associated inherited optic neuropathies. In addition, these data reinforce the concept that mutation in either OPA1 intronic sequences involved in splicing regulation or in genes coding for splicing effectors could potentially be pathogenic.

\section{Materials and Methods}

Antibodies. OPA1 antibodies were described previously. ${ }^{7}$ Commercial antibodies were from the sources indicated: monoclonal anti-cyt $c$ (Promega), polyclonal anti-cleaved PARP (Promega), monoclonal anti-actin (Chemicon), Alexa594 anti-rabbit IgG and Alexa-488 anti-mouse IgG (Molecular Probes), anti-rabbit IgG-HRP and anti-mouse lgG-HRP (New England Biolabs).
Plasmid constructions, oligonucleotides and siRNA sequences, and Q-PCR. OPA1 variants were cloned by RT-PCR using oligonucleotides situated in the Ex3 and Ex5 to amplify sequences either containing or not Ex4 and Ex4b, and oligonucleotides situated in Ex5 and Ex9 to amplify sequences either containing or not Ex5b. All amplified fragments were cloned in the topo-pCRIl vector (Invitrogen), sequenced and recombined in OPA1 open reading frame to provide the four transcripts OPA1-0, OPA1-4, OPA1-4b and OPA1-5b. They were further subcloned in the PREP41 and PCCEY plasmids.

Oligonucleotide sequences to perform Q-PCR were: SEx3-5, 5'-GAGTATATC GATTTTGGTTCTC-3'; SEx4-5; $5^{\prime}$-CTTTTTTACCTCAGGTTCTCC-3'; SEX3-4b; 5'-GAGTATATCGATTTTGGTCACA-3'; SEx4-4b; 5'-GACTTTTTTACCTCAGGT CAC-3'; ASEx5-6; 5'-CTCTTTGTCTGACACCTTTCT-3'; and ASEx5-5b; 5'TCACCAAGCAGACCCTTTCT- $3^{\prime}$. cDNAs were synthesized from HeLa cell total RNA or from commercial human organ RNAs (Clontech) using the Superscript $\AA$ II reverse transcriptase (Invitrogen). Triplicate Q-PCR were performed using the four different sense primers and the two antisens primers, and the Master plus SYBR Green I kit, in a LightCyclerß FastStart DNA devise (Roche Applied Science). Q-PCR products were systematically checked on $2 \%$ agarose gels. siRNA (Dharmacond Research) exon-specific sequences are 5'-AAGATTGTT GAAAGCCTTAGCTT-3' for Ex4, 5'-AAGTCATAGGAGCTTCTGACCTA-3' for Ex4b and $5^{\prime}$-AAGAGGAAGCGCGCAGAGCCGCT-3' for Ex5b.

Fission yeast complementation test, HeLa cell culture and transfection, and Western blots. Diploid fission yeast strain deleted for msp1 were transformed by the pREP41-OPA1-(0, 4, 4b or $5 \mathrm{~b})$ or pREP41-msp1 plasmids, then induced to sporulate. Five thousand spores from each transformation were spotted on EMMA medium to select for haploid cells deleted for $m s p 1$ and expressing the different OPA1 variant. ${ }^{34}$

HeLa cells were cultured in DMEM, $10 \% \mathrm{FCS}$ and $5 \% \mathrm{CO}_{2}$. Transfections of HeLa cells were performed with Lipofectamine $2000 \AA$ reagent (Invitrogen). siRNA (Dharmacond Research) experiments were carried out as described previously with some modifications. ${ }^{11,35}$ Final concentration of the siRNA duplex in culture medium was $100 \mathrm{nM}$. Inhibition of caspases was obtained with $100 \mu \mathrm{M}$ z-VAD-fmk (Calbiochem). To detect OPA1 and actin by Western blots, transfected cells were harvested, washed once in PBS and equal amounts of proteins were solubilized in $50 \mu \mathrm{l}$ Laemelli sample buffer and boiled for $10 \mathrm{~min}$. Samples were run on $7 \%$ polyacrylamide gels and transferred to nitrocellulose. Immunodetection (anti-OPA1: 1/2000 for overexpression experiments and 1/400 for RNAi; anti-actin: 1/10000; IgG-HRP: 1/10000) was carried out using ECL (Amersham Pharmacia Biotech). PARP detection was performed according to the protocol from Promega (antiPARP: $1 / 400)$.

Microscopy. For indirect immunofluorescent microscopy, cells grown on glass coverslips were fixed in PBS, $3.7 \%$ paraformaldehyde $\left(30 \mathrm{~min}, 4^{\circ} \mathrm{C}\right)$, permeabilized in PBS, $0.2 \%$ Triton X-100 (10 min, room temperature) and immuno-labelled in PBS, $2 \%$ BSA, using the following antibodies (OPA1: 1/800; cyt c: 1/1000; Alexa-594 anti-rabbit IgG: 1/600; Alexa-488 anti-mouse IgG: 1/600) and stained with DAPI $(0.1 \mu \mathrm{g} / \mathrm{ml})$. For direct labelling procedure of mitochondria and chromatin, cell cultures were stained using $100 \mathrm{nM}$ CMXros Mitotracker $($ Red (Molecular Probes) for $30 \mathrm{~min}$, then fixed and DAPI stained. In all experiments, phenotypes were counted on 300 cells in duplicate or triplicate experiments. Statistics were performed using the one way ANOVA test, followed by the Dunnett test with $P<0.05$. To analyse the $\Delta \Psi_{\mathrm{m}}$, cells were incubated $20 \mathrm{~min}$ with $5 \mu \mathrm{g} / \mathrm{ml} \mathrm{JC}-1$ (Molecular Probes) in culture medium and either directly observed in microscopy in culture medium without phenol red (Gibco) or subsequently collected, washed in PBS and processed for fluorescence analysis on an Ascent Fluoroscan using the Ascent Software (excitation $488 \mathrm{~nm}$; emission 538 and $590 \mathrm{~nm}$ ). The ratio of the fluorescence at $590 \mathrm{~nm}$, proportional to the $\Delta \Psi_{\mathrm{m}}$, to that at $538 \mathrm{~nm}$, proportional to the mass of mitochondria, was calculated from triplicate values obtained in the course of three independent experiments. Fluorescence images were acquired and processed using a Leica DMIRE-2 microscope.

For TEM, cells were transfected either by siRNA and analysed $72 \mathrm{~h}$ afterwards or microinjected using a Zeiss Axiovert microinjector with the different plasmids in the presence of anti-rabbit antibodies conjugated to $10 \mathrm{~nm}$ gold beads (EMS) and harvested after $24 \mathrm{~h}$. Cells were fixed for $2 \mathrm{~h}$ with $4 \%$ glutaraldehyde in sodium cacodylate buffer, post-fixed for $1 \mathrm{~h}$ with $1 \%$ osmium tetroxide, dehydrated and embedded in Epon (EMS). Thin sections collected onto nickel grids were stained with $1 \%$ uranyl acetate and $0.3 \%$ lead citrate, and observed in a JEOL-1200 EX electron microscope at $80 \mathrm{kV}$. 
Acknowledgements. We are indebted to the IFR 76 and IFR 109 facilities, to Nacer Benméragui for technical help in electron microscopy, to Martine Cazales for technical help for microinjections and to members of the INSERM U583 and CNRS UMR5088 for helpful discussions and comments on the paper. This work was supported by grants from CNRS, INSERM, Rétina France, Fondation pour la Recherche Médicale, Fondation Electricité de France and l'Association Française contre les Myopathies. AO was a recipient of an ARC fellowship and GE recipient of an UNADEV fellowship.

1. Perfettini JL, Roumier T, Kroemer G. Mitochondrial fusion and fission in the control of apoptosis. Trends Cell Biol 2005; 15: 179-183.

2. Youle RJ, Karbowski M. Mitochondrial fission in apoptosis. Nat Rev Mol Cell Biol 2005; 6 : 657-663.

3. Scorrano L, Ashiya M, Buttle K, Weiler S, Oakes SA, Mannella CA et al. A distinct pathway remodels mitochondrial cristae and mobilizes cytochrome $c$ during apoptosis. Dev Cell 2002; 2: 55-67.

4. Santel A, Fuller MT. Control of mitochondrial morphology by a human mitofusin. J Cell Sci 2001; 114: 867-874.

5. Eura Y, Ishihara N, Yokota S, Mihara K. Two mitofusin proteins, mammalian homologues of FZO, with distinct functions are both required for mitochondrial fusion. $J$ Biochem (Tokyo) 2003; 134: 333-344.

6. Smirnova E, Griparic L, Shurland DL, van der Bliek AM. Dynamin-related protein Drp1 is required for mitochondrial division in mammalian cells. Mol Biol Cell 2001; 12: 2245-2256.

7. Olichon A, Emorine LJ, Descoins E, Pelloquin L, Brichese L, Gas $\mathrm{N}$ et al. The human dynamin-related protein OPA1 is anchored to the mitochondrial inner membrane facing the inter-membrane space. FEBS Lett 2002; 523: 171-176.

8. Karbowski M, Lee YJ, Gaume B, Jeong SY, Frank S, Nechushtan A et al. Spatial and temporal association of Bax with mitochondrial fission sites, Drp1, and Mfn2 during apoptosis. J Cell Biol 2002; 159: 931-938.

9. Germain M, Mathai JP, McBride HM, Shore GC. Endoplasmic reticulum BIK initiates DRP1-regulated remodelling of mitochondrial cristae during apoptosis. EMBO J 2005; 24 : 1546-1556.

10. Legros F, Lombes A, Frachon P, Rojo M. Mitochondrial fusion in human cells is efficient, requires the inner membrane potential, and is mediated by mitofusins. Mol Biol Cell 2002; 13: 4343-4354.

11. Olichon A, Baricault L, Gas N, Guillou E, Valette A, Belenguer $P$ et al. Loss of OPA1 perturbates the mitochondrial inner membrane structure and integrity, leading to cytochrome $c$ release and apoptosis. J Biol Chem 2003; 278: 7743-7746.

12. Cipolat S, Martins de Brito O, Dal Zilio B, Scorrano L. OPA1 requires mitofusin 1 to promote mitochondrial fusion. Proc Natl Acad Sci USA 2004; 101: 15927-15932.

13. Frey TG, Mannella CA. The internal structure of mitochondria. Trends Biochem Sci 2000; 25: 319-324.

14. Frezza C, Cipolat S, Martins de Brito O, Micaroni M, Beznoussenko GV, Rudka T et al. OPA1 controls apoptotic cristae remodeling independently from mitochondrial fusion. Cell 2006; 126: 177-189.

15. Chen $\mathrm{H}$, Chomyn A, Chan DC. Disruption of fusion results in mitochondrial heterogeneity and dysfunction. J Biol Chem 2005; 280: 26185-26192.

16. Arnoult D, Grodet A, Lee YJ, Estaquier J, Blackstone C. Release of OPA1 during apoptosis participates in the rapid and complete release of cytochrome $C$ and subsequent mitochondrial fragmentation. J Biol Chem 2005; 280: 35742-35750.
17. Griparic L, van der Wel NN, Orozco IJ, Peters PJ, van der Bliek AM Loss of the intermembrane space protein Mgm1/OPA1 induces swelling and localized constrictions along the lengths of mitochondria. J Biol Chem 2004; 279: 18792-18798.

18. Lee YJ, Jeong SY, Karbowski M, Smith CL, Youle RJ. Roles of the mammalian mitochondrial fission and fusion mediators Fis1, Drp1, and Opa1 in apoptosis. Mol Biol Cell 2004; 15: 5001-5011.

19. Satoh M, Hamamoto T, Seo N, Kagawa Y, Endo H. Differential sublocalization of the dynamin-related protein OPA1 isoforms in mitochondria. Biochem Biophys Res Commun 2003; 300: 482-493

20. Delettre C, Griffoin JM, Kaplan J, Dollfus H, Lorenz B, Faivre L et al. Mutation spectrum and splicing variants in the OPA1 gene. Hum Genet 2001; 109: 584-591.

21. Ishihara N, Fujita Y, Oka T, Mihara K. Regulation of mitochondrial morphology through proteolytic cleavage of OPA1. EMBO J 2006; 25: 2966-2977.

22. Cipolat S, Rudka T, Hartmann D, Costa V, Serneels L, Craessaerts K et al. Mitochondrial rhomboid PARL regulates cytochrome $c$ release during apoptosis via OPA1-dependent cristae remodeling. Cell 2006; 126: 163-175.

23. Ferre M, Amati-Bonneau $\mathrm{P}$, Tourmen $\mathrm{Y}$, Malthiery $\mathrm{Y}$, Reynier $\mathrm{P}$. eOPA1: an online database for OPA1 mutations. Hum Mutat 2005; 25: 423-428.

24. Wong ED, Wagner JA, Scott SV, Okreglak V, Holewinske TJ, Cassidy-Stone A et al. The intramitochondrial dynamin-related GTPase, Mgm1p, is a component of a protein complex that mediates mitochondrial fusion. J Cell Biol 2003; 160: 303-311.

25. Guillou E, Bousquet C, Daloyau M, Emorine LJ, Belenguer P. Msp1p is an intermembrane space dynamin-related protein that mediates mitochondrial fusion in a Dnm1p-dependen manner in S. pombe. FEBS Lett 2005; 579: 1109-1116.

26. Herlan M, Vogel F, Bornhvd C, Neupert W, Reichert AS. Processing of MGM1 by the rhomboid-type protease Pcp1 is required for maintenance of mitochondrial morphology and of mitochondrial DNA. J Biol Chem 2003; 278: 27781-27788.

27. McQuibban GA, Saurya S, Freeman M. Mitochondrial membrane remodelling regulated by a conserved rhomboid protease. Nature 2003; 423 : 537-541.

28. Delettre C, Lenaers G, Griffoin JM, Gigarel N, Lorenzo C, Belenguer $P$ et al. Nuclear gene OPA1, encoding a mitochondrial dynamin-related protein, is mutated in dominant optic atrophy. Nat Genet 2000; 26: 207-210.

29. Tondera D, Czauderna F, Paulick K, Schwarzer R, Kaufmann J, Santel A. The mitochondrial protein MTP18 contributes to mitochondrial fission in mammalian cells. J Cell Sci 2005; 118: 3049-3059.

30. John GB, Shang Y, Li L, Renken C, Mannella CA, Selker JM et al. The mitochondrial inner membrane protein mitofilin controls cristae morphology. Mol Biol Cell 2005; 16 : 1543-1554.

31. Westermann B. Merging mitochondria matters: cellular role and molecular machinery of mitochondrial fusion. EMBO Rep 2002; 3: 527-531.

32. Yu T, Fox RJ, Burwell LS, Yoon Y. Regulation of mitochondrial fission and apoptosis by the mitochondrial outer membrane protein hFis1. J Cell Sci 2005; 118 4141-4151.

33. Clayton R, Clark JB, Sharpe M. Cytochrome $c$ release from rat brain mitochondria is proportional to the mitochondrial functional deficit: implications for apoptosis and neurodegenerative disease. J Neurochem 2005; 92: 840-849.

34. Lenaers G, Pelloquin L, Olichon A, Emorine LJ, Guillou E, Delettre C et al. What similarity between human and fission yeast proteins is required for orthology? Yeast 2002; 19: $1125-1126$.

35. Elbashir SM, Harborth J, Weber $\mathrm{K}$, Tuschl T. Analysis of gene function in somatic mammalian cells using small interfering RNAs. Methods 2002; 26: 199-213.

\section{Supplementary Information accompanies the paper on Cell Death and Differentiation website (http://www.nature.com/cdd)}

